\title{
THE ASYMPTOTIC BEHAVIOUR OF CERTAIN INTEGRAL FUNCTIONS
}

\author{
BY
}

P. C. FENTON

ABSTRACT. Let $f(z)$ be an integral function satisfying

$$
\int^{\infty}\{\log m(r, f)-\cos \pi \rho \log M(r, f)\}^{+} \frac{d r}{r^{\rho+1}}<\infty
$$

and

$$
0<\varliminf_{r \rightarrow \infty} \frac{\log M(r, f)}{r^{\rho}}<\infty
$$

for some $\rho: 0<\rho<1$. It is shown that such functions have regular asymptotic behaviour outside a set of circles with centres $\zeta_{i}$ and radii $t_{i}$ for which

$$
\sum_{i=1}^{\infty} \frac{t_{i}}{\left|\zeta_{i}\right|}<\infty
$$

1. Introduction. For an integral function $f(z)$ let

$$
M(r, f)=\max _{|z|=r}|f(z)|, \quad m(r, f)=\min _{|z|=r}|f(z)|
$$

and let $n(r, f)$ be the number of zeros of $f$ in $|z| \leqslant r$. The order $\rho$ of $f$ is

$$
\rho=\varlimsup_{r \rightarrow \infty} \frac{\log \log M(r, f)}{\log r} .
$$

The following result appears in [6].

THEOREM A. Let $\rho$ be a positive number less than one and let $f(z)$ be an integral function of order $\rho$ satisfying the following conditions:

(i) there is a finite constant $K$ such that

$$
\varlimsup_{\substack{r_{2}>r_{1} \\ r_{1} \rightarrow \infty}} \int_{r_{1}}^{r_{2}}\{\log m(r, f)-\cos \pi \rho \log M(r, f)\} \frac{d r}{r^{\rho+1}} \leqslant K ;
$$

(ii) there are numbers $\alpha$ and $\beta$, with $0<\alpha<\beta<\infty$, such that, for all large $r$,

$$
\alpha r^{\rho} \leqslant n(r, f) \leqslant \beta r^{\rho} .
$$

Let

Received by the editors March 28, 1976 and, in revised form, April 1, 1977. AMS (MOS) subject classifications (1970). Primary 30A64. 


$$
k=\left\{\frac{2 \beta}{\alpha}\right\}^{1 / \rho} \text {. }
$$

Then there is a curve $C: z=r e^{i \phi(r)}$, where $\phi(r)$ is a continuous function satisfying

$$
\left|\phi\left(R_{1}\right)-\phi\left(R_{2}\right)\right|=o\left|\log \frac{R_{2}}{R_{1}}\right|^{1 / 2} \text { as } \min \left(R_{1}, R_{2}\right) \rightarrow \infty,
$$

a function $\varepsilon(t)$ satisfying $\pi \geqslant \varepsilon(t) \geqslant 0$ and $\varepsilon(t) \rightarrow 0$ as $t \rightarrow \infty$, and a function $\nu(t)$ satisfying $1 \geqslant \nu(t) \geqslant 0, \nu(t) \rightarrow 0$ as $t \rightarrow \infty$ and

$$
\int^{\infty} \frac{\nu(t)}{t} d t<\infty
$$

for which the following is true. If $\zeta$ is any point on $C$, then the set

$$
\left\{z: k^{-1}|\zeta|<|z| \leqslant k|\zeta| \text { and }\left|\arg z \zeta^{-1}\right|>\varepsilon(|\zeta|)\right\}
$$

contains at most $\nu(|\zeta|) N(|\zeta|)$ zeros of $f$, where $N(|\zeta|)$ is the number of zeros of $f$ in

$$
\left\{z: k^{-1}|\zeta|<|z| \leqslant k|\zeta|\right\}
$$

The equation (1.2) is a consequence of the following: there is a constant $A=A(k)$ and a function $\Delta(t)$ satisfying $\pi \geqslant \Delta(t) \geqslant 0, \Delta(t) \rightarrow 0$ and

$$
\int^{\infty} \frac{\Delta(t)^{2}}{t} d t<\infty
$$

for which

$$
\left|\phi^{\prime}(t)\right| \leqslant A \frac{\Delta(t)}{t} \text { for all large } t .
$$

The reader is referred to [6] for details.

It will be shown here that this result leads to a precise description (outside a small exceptional set) of the asymptotic behavior of a certain class of integral functions. To be specific, let $\rho$ be a positive number less than one and suppose that $f$ is an integral function satisfying

(i)' with the convention that $a^{+}=\max (0, a)$ for any real number $a$,

$$
\int^{\infty}\{\log m(r, f)-\cos \pi \rho \log M(r, f)\}^{+} \frac{d r}{r^{\rho+1}}<\infty ;
$$

(ii)' there is a finite nonzero constant $\beta$ such that

$$
0<\beta=\lim _{r \rightarrow \infty} \frac{\log M(r, f)}{r^{\rho}}<\infty .
$$

We shall prove here 
THEOREM 1. Let $\rho$ be a positive number less than one and let $f(z)$ be an integral function satisfying conditions (i)' and (ii)' above. Then $f(z)$ satisfies the hypotheses of Theorem $\mathrm{A}$ and with $\phi(r)$ as in that theorem we have

$$
\left|r^{-\rho} \log \right| f\left(r e^{i(\phi(r)+\theta-\pi)}\right)|-\beta \cos \rho \theta|=o(1)
$$

as $r$ tends to infinity outside a set of discs with centres $\zeta_{i}$, radii $t_{i}$, for which

$$
\sum_{i=1}^{\infty} \frac{t_{i}}{\left|\zeta_{i}\right|}<\infty
$$

The exceptional set of Theorem 1 may be described briefly, following Hayman [7], as an E-set. Theorem 1 has much in common with results of Essén [4] and Essén and Lewis [5] on subharmonic functions. In [4] Essén is concerned with functions subharmonic in the plane slit along the negative real axis while [5] generalizes the considerations of [4] to functions subharmonic in $d$-dimensional cones and also establishes an improved estimate of the exceptional set. When restricted to integral functions the result of [4] combined with the estimate of the exceptional set of [5] may be viewed as a special case of Theorem 1, when $|f(r)|=M(r, f),|f(-r)|=m(r, f)$ and $\log |f(-r)| \leqslant \cos \pi \rho \log |f(r)|$.

The condition (i)' cannot be replaced with

$$
\varlimsup_{r_{1}, r_{2} \rightarrow \infty} \int_{r_{1}}^{r_{2}}\{\log m(r, f)-\cos \pi \rho \log M(r, f)\} \frac{d r}{r^{\rho+1}} \leqslant 0,
$$

a condition arising in the work of Anderson [1]. For in [1], Anderson shows that

$$
\int_{0}^{\infty}\{\log |f(-r)|-\cos \pi \rho \log |f(r)|\} \frac{d r}{r^{\rho+1}}
$$

exists (so that.(1.8) certainly holds) for an integral function $f(z)$ with real negative zeros if

$$
\frac{\log f(r)}{r^{\rho}} \rightarrow A \quad(0<A<\infty)
$$

for some $\rho: 0<\rho<1$. It will be shown in $\$ 9$, however, that there exists an integral function $f(z)$ with real negative zeros satisfying (1.9) and such that, for some $\varepsilon>0$,

$$
\log |f(-r)|<(A \cos \pi \rho-\varepsilon) r^{\rho}
$$

for all $r$ in a set of infinite logarithmic measure. Since an $E$-set intersects every ray through the origin in a set of finite logarithmic measure (1.6) cannot hold outside an $E$-set. 
2. Preliminaries. From (i)' it follows that

$$
\varlimsup_{r_{1}, r_{2} \rightarrow \infty} \int_{r_{1}}^{r_{2}}\{\log m(r, f)-\cos \pi \rho \log M(r, f)\} \frac{d r}{r^{\rho+1}} \leqslant 0
$$

and from this together with (ii) ${ }^{\prime}$ and the theorem of Anderson already mentioned [1, p. 154] we deduce that

$$
\begin{aligned}
\log M(r, f) & \sim \beta r^{\rho}, \\
\log f_{1}(r) & \sim \beta r^{\rho},
\end{aligned}
$$

where

$$
f_{1}(z)=\prod_{1}^{\infty}\left(1+\frac{z}{\left|a_{n}\right|}\right)
$$

the numbers $a_{n}, n=1,2,3, \ldots$, being the nonzero zeros of $f$ arranged in order of increasing magnitude. A well-known consequence of (2.2) is that

$$
n(r, f)=n\left(r, f_{1}\right) \sim \pi^{-1} \beta \sin \pi \rho r^{\rho},
$$

so that functions satisfying (i) ${ }^{\prime}$ and (ii) ${ }^{\prime}$ are of order $\rho$ and satisfy (i) and (ii) of Theorem A.

In the course of the proof of Theorem 1 we shall find it convenient to refer to a result due to Kolomiiceva [9]. A complete discussion of Kolomiiceva's theorem would involve us in needless complications but a simple consequence of it is

LEMMA 1. Let $g(z)$ be an integral function satisfying

$$
\lim _{r \rightarrow \infty} \frac{\log M(r, g)}{r^{\rho}}=\beta
$$

where $0<\rho<1$ and $0<\beta<\infty$, which is such that, for each $\eta>0$, the number of zeros of $g$ in

$$
\{|z| \leqslant r\} \cap\{|\arg z| \leqslant \pi-\eta\}
$$

is $o\left(r^{\rho}\right)$ as $r \rightarrow \infty$. Then a necessary and sufficient condition that

$$
\log \left|g\left(r e^{i \theta}\right)\right|=(\beta \cos \rho \theta+o(1)) r^{\rho}
$$

outside a set $E$ is the following: given $\varepsilon>0$, there exist $\delta=\delta(\varepsilon)>0$ and $r(\varepsilon)$ such that for all $z$ outside E satisfying $|z|>r(\varepsilon)$,

$$
\int_{0}^{\delta r} \frac{n_{z}(t, g)}{t} d t<\varepsilon r^{\rho},
$$

where $n_{z}(t, g)$ is the number of zeros of $g$ contained in the open disc with centre $z$ and radius $t$. 
3. An auxiliary function. We suppose without loss of generality that $f(0)=1$ so that

$$
f(z)=\prod_{1}^{\infty}\left(1-\frac{z}{a_{n}}\right)
$$

As was mentioned before the results of Theorem A hold for functions satisfying the hypotheses of Theorem 1. Choose

$$
k=\left(\begin{array}{ll}
1 & 2
\end{array}\right)^{1 / \rho}
$$

and let $C$ and $\phi(t)$ be as in Theorem A. We relabel $C$ as $C_{\pi}$ and for every $\theta$ satisfying $-\pi<\theta<\pi$ we define $C_{\theta}$ by

$$
C_{\theta}: z=r e^{i(\phi(r)+\theta-\pi)} .
$$

Let us rearrange the zeros of $f$ in the following way: if $a_{n}$ is a zero of $f$ lying on the curve $C_{\theta}$ say, we transfer it to the point $a_{n}^{\prime}=\left|a_{n}\right| e^{i \theta}$ and define

$$
F(z)=\prod_{1}^{\infty}\left(1-\frac{z}{a_{n}^{\prime}}\right)
$$

Our first concern is to show that $\log \left|F\left(|z| e^{i \theta}\right)\right|$ and $\log |f(z)|$ do not greatly differ. Later we shall show that $\log |F(z)|$ and $\log \left|f_{1}(z)\right|$ have similar asymptotic behavior and then, after estimating $\log \left|f_{1}(z)\right|$, we shall appeal to the intermediate character of $F$ to estimate $\log |f(z)|$.

4. Comparison of $f$ and $F$. We shall prove

LEMMA 2. Given any number $\varepsilon>0$, there exists a number $R(\varepsilon)$ such that, if $f(z) \neq 0$,

$$
|\log | f(z)|-\log | F\left(|z| e^{i \theta}\right)||<\varepsilon r^{\rho}
$$

whenever $|z|>R(\varepsilon)$, where $\theta$ satisfies $-\pi<\theta \leqslant \pi$ and is such that $z$ lies on $C_{\theta}$.

Throughout the proof we suppose that $z=r e^{i \psi}$ is not a zero of $f$. We have, from (3.1) and (3.4),

$$
\log \left|\frac{f(z)}{F\left(r e^{i \theta}\right)}\right|=\sum_{1}^{\infty} \log \left|\left(1-\frac{z}{a_{n}}\right)\left(1-\frac{r e^{i \theta}}{a_{n}^{\prime}}\right)^{-1}\right|
$$

and we examine the sum of (4.2) in three parts. First, with $a_{n}=r_{n} e^{i \phi_{n}}$ consider, for $p>1$, 


$$
\begin{aligned}
S_{1} & =\prod_{r_{n}>k^{p_{r}}} \log \left|\left(1-\frac{z}{a_{n}}\right)\left(1-\frac{r e^{i \theta}}{a_{n}^{\prime}}\right)^{-1}\right| \\
& \leqslant \sum_{r_{n}>k^{p_{r}}} \log \left(1+\frac{r}{r_{n}}\right)\left(1-\frac{r}{r_{n}}\right)^{-1} \\
& \leqslant 2 r\left(1-k^{-1}\right)^{-1} \sum_{r_{n}>k^{p_{r}}} r_{n}^{-1} \\
& =2 r\left(1-k^{-1}\right)^{-1} \int_{k^{p_{r}}}^{\infty} \frac{d n(t)}{t} .
\end{aligned}
$$

Integrating by parts we obtain

$$
S_{1}=O\left(k^{p(\rho-1)} r^{\rho}\right)
$$

Next consider

$$
\begin{aligned}
S_{2} & =\sum_{r_{n}<k^{-p_{r}}} \log \left|\left(1-\frac{z}{a_{n}}\right)\left(1-\frac{r e^{i \theta}}{a_{n}^{\prime}}\right)^{-1}\right| \\
& \leqslant \sum_{r_{n}<k^{-p_{r}}} \log \left\{\left(1+\frac{r_{n}}{r}\right)\left(1-\frac{r_{n}}{r}\right)^{-1}\right\} \\
& \leqslant 2 r^{-1}\left(1-k^{-1}\right)^{-1} \sum_{r_{n}<k^{-p_{r}}} r_{n} \\
& =2 r^{-1}\left(1-k^{-1}\right)^{-1} \int_{0}^{k^{-p_{r}}} t d n(t) \\
& =O\left(k^{-p(\rho+1)} r^{\rho}\right) .
\end{aligned}
$$

Finally we consider the remaining part of the sum, that for which $k^{-p_{r}} \leqslant$ $r_{n} \leqslant k^{p} r$. Since $\theta=\pi+\psi-\phi(r)$ and $a_{n}^{\prime}=r_{n} e^{i\left(\pi+\phi_{n}-\phi\left(r_{n}\right)\right)}$,

$$
\begin{aligned}
J_{n} & =\left|\left(1-\frac{z}{a_{n}}\right)\left(1-\frac{r e^{i \theta}}{a_{n}^{\prime}}\right)^{-1}\right|^{2} \\
& =\frac{\left(1-\frac{r}{r_{n}}\right)^{2}+\frac{4 r}{r_{n}} \sin ^{2}\left(\frac{\psi-\phi_{n}}{2}\right)}{\left(1-\frac{r}{r_{n}}\right)^{2}+\frac{4 r}{r_{n}} \sin ^{2}\left(\frac{\psi-\phi_{n}-\phi(r)+\phi\left(r_{n}\right)}{2}\right)}
\end{aligned}
$$

Let us write $t_{n}=r / r_{n}, \psi-\phi_{n}=\psi_{n}, \phi(r)-\phi\left(r_{n}\right)=\nu_{n}$. Then 


$$
\begin{aligned}
J_{n} & =\frac{\left(1-t_{n}\right)^{2}+4 t_{n} \sin ^{2}\left(\psi_{n} / 2\right)}{\left(1-t_{n}\right)^{2}+4 t_{n} \sin ^{2}\left(\left(\psi_{n}-\nu_{n}\right) / 2\right)} \\
& =1+\frac{4 t_{n} \sin \left(\psi_{n}-\frac{1}{2} \nu_{n}\right) \sin \frac{1}{2} \nu_{n}}{\left(1-t_{n}\right)^{2}+4 t_{n} \sin ^{2}\left(\left(\psi_{n}-\nu_{n}\right) / 2\right)} .
\end{aligned}
$$

Hence

$$
\begin{aligned}
\log J_{n} \leqslant & \frac{4 t_{n}\left|\sin \left(\psi_{n}-\frac{1}{2} \nu_{n}\right) \sin \frac{1}{2} \nu_{n}\right|}{\left(1-t_{n}\right)^{2}+4 t_{n} \sin ^{2}\left(\frac{\psi_{n}-\nu_{n}}{2}\right)} \\
& \leqslant \frac{8 t_{n}\left|\sin \left(\frac{\psi_{n}-\nu_{n}}{2}\right) \sin \frac{1}{2} \nu_{n}\right|+8 t_{n}\left|\sin \frac{1}{4} \nu_{n} \sin \frac{1}{2} \nu_{n}\right|}{\left(1-t_{n}\right)^{2}+4 t_{n} \sin ^{2}\left(\frac{\psi_{n}-\nu_{n}}{2}\right)}
\end{aligned}
$$

since, for any real numbers $a$ and $b$,

$$
\begin{aligned}
\left|\sin \left(a-\frac{1}{2} b\right)\right| & \leqslant 2\left|\sin \left(\frac{1}{2}(a-b)+\frac{1}{4} b\right)\right| \\
& \leqslant 2\left|\sin \frac{1}{2}(a-b)\right|+2\left|\sin \frac{1}{4} b\right| .
\end{aligned}
$$

Further, from (1.5),

$$
\begin{aligned}
\left|\nu_{n}\right| & =\left|\phi(r)-\phi\left(r_{n}\right)\right| \leqslant\left|\int_{r_{n}}^{r}\right| \phi^{\prime}(t)|d t| \\
& \leqslant\left|\int_{r_{n}}^{r} A \frac{\Delta(t)}{t} d t\right| \leqslant A\left|\log \frac{r}{r_{n}}\right|\left\{\sup _{t>k^{-p_{r}}} \Delta(t)\right\} \\
& \leqslant A k^{p}\left|1-\frac{r}{r_{n}}\right|\left\{\sup _{t>k^{-p_{r}}} \Delta(t)\right\} .
\end{aligned}
$$

Substituting (4.6) into (4.5) we obtain

$$
\begin{aligned}
\log J_{n} \leqslant & \frac{4 A k^{p_{t}}\left|1-t_{n}\right|\left|\sin \left(\frac{\psi_{n}-\nu_{n}}{2}\right)\right|}{\left(1-t_{n}\right)^{2}+4 t_{n} \sin ^{2}\left(\frac{\psi_{n}-\nu_{n}}{2}\right)}\left\{\sup _{t>k^{-p_{r}}} \Delta(t)\right\} \\
& +A^{2} k^{2 p_{t_{n}}}\left\{\sup _{t>k^{-p_{r}}} \Delta(t)^{2}\right\} \\
\leqslant & A k^{p^{2} t_{n}^{1 / 2}}\left\{\sup _{t>k^{-p_{r}}} \Delta(t)\right\}+A^{2} k^{2 p_{t_{n}}}\left\{\sup _{t>k^{-p_{r}}} \Delta(t)^{2}\right\} \\
\leqslant & A_{1} k^{3 p}\left\{\sup _{t>k^{-p_{r}}} \Delta(t)\right\},
\end{aligned}
$$


where $A_{1}=A+\pi A^{2}$. Hence, since from (2.4) the number of zeros of $f$ in $|z| \leqslant k^{p} r$ is at most $2 \alpha k^{p \rho} r^{\rho}$ for large $r$, where $\alpha=\beta \pi^{-1} \sin \pi \rho$,

$$
\begin{aligned}
S_{3} & =\sum_{k^{-p_{r}}<r_{n}<k^{p_{r}}} \log \left|\left(1-\frac{z}{a_{n}}\right)\left(1-\frac{r e^{i \theta}}{a_{n}^{\prime}}\right)^{-1}\right| \\
& \leqslant 2 \alpha A_{1} k^{p(\rho+3)} r^{\rho}\left\{\sup _{t>k^{-p_{r}}} \Delta(t)\right\} .
\end{aligned}
$$

Given $\varepsilon>0$ we may choose $p$ sufficiently large that $S_{1}+S_{2}<\varepsilon r^{\rho}$ for all large $r$ and with this $p$ we may choose $r_{0}(\varepsilon)$ so that $S_{3}<\varepsilon r^{\rho}$ for $r>r_{0}(\varepsilon)$, since $\Delta(t) \rightarrow 0$ as $t \rightarrow \infty$, which proves one half of Lemma 1 . The second half, that

$$
\log \left|\frac{F\left(|z| e^{i \theta}\right)}{f(z)}\right|<\varepsilon|z|^{\rho},
$$

is proved similarly.

5. The zeros of $F(z)$. We shall prove

LeMma 3. Let $\delta$ be a fixed positive number less than $\pi$ and let $n_{z}(t, F, \delta)$ be the number of zeros of $F$ contained in

$$
\left\{\zeta:|\arg \zeta|<\pi-\frac{1}{2} \delta\right\} \cap\{\zeta:|\zeta-z|<t\} .
$$

Then given any positive number $\varepsilon<\frac{1}{2}$ there exists a number $R(\varepsilon, \delta)$ such that, with $|z|=r$,

$$
\int_{0}^{e r} n_{z}(t, F, \delta) \frac{d t}{t}<\varepsilon r^{\rho}
$$

for all $z$ outside a set $H_{1}$ (where $H_{1}$ depends only on $\delta$ ) and such that $|z|>R(\varepsilon, \delta)$. Moreover $H_{1}$ is covered by a set of discs $C_{i}$, centres $\zeta_{i}$, radii $t_{i}$, $i=1,2,3, \ldots$, such that $\Sigma_{1}^{\infty} t_{i} /\left|\zeta_{i}\right|<\infty$.

Throughout the proof of Lemma 3 we write $n_{z}(t), n_{z}(t, \delta)$ instead of $n_{z}(t, F), n_{z}(t, F, \delta)$.

We shall make use of an argument of Azarin [2] in which the following lemma is used.

Lemma 4 ([10, Lemma 3.2]). If a set $E$ in the complex plane is covered by discs of bounded radii such that each point of the set is the centre of a disc, then from this one may select a subsystem of discs which covers the set, each point of the plane being covered no more than $\nu$ times by the discs of this subsystem, where $\nu$ is an absolute constant.

Let $R_{1}=R_{1}(\delta)$ be such that, for $r \geqslant R_{1}$ we have $\varepsilon(r)<\frac{1}{2} \delta$, where $\varepsilon(r)$ is 
the function occurring in Theorem A. (We may note that, if $z \in S\left(\delta, R_{1}\right)$, where

$$
S\left(\delta, R_{1}\right)=\left\{z:|z| \geqslant R_{1} \text { and }\{\arg z \mid \leqslant \pi-\delta\},\right.
$$

then $n_{z}(t, \delta)=n_{z}(t)$ certainly for $0<t<\frac{1}{4} \delta|z|$.) Let $H_{1}$ be the set of points $z$ in $|z| \geqslant R_{1}$ at which, for some $t=t(z)$ satisfying $0<t<\frac{1}{2}|z|$, we have

$$
n_{z}(t, \delta) \geqslant t|z|^{\rho-1} \text {. }
$$

Let $E_{n}$ be the subset of $H_{1}$ contained in the annulus

$$
\left\{z: 4^{n+1}>|z| \geqslant 4^{n}\right\}, \quad n=0,1,2, \ldots
$$

We surround each point $z$ of $H_{1}$ by a disc of radius $t(z)$ and from the set of such discs surrounding points of $E_{n}$ we select a subsystem $K_{n}$ which covers $E_{n}$, while covering each point of the plane at most $\nu$ times. This can be done, by Lemma 4 . We note that the members of $K_{m}$ do not intersect the members of $K_{n}$ if $|n-m| \geqslant 2$, and therefore $K=\cup_{n=1}^{\infty} K_{n}$ is a set of discs the members of which cover each point of the plane at most $2 \nu$ times.

Now, $K$ is a countable set the members of which may be ordered: $C_{i}$, $i=1,2,3, \ldots$, where $C_{i}$ is a disc with centre $\zeta_{i}$ and radius $t_{i}$, where $0<t_{i}<\frac{1}{2}\left|\zeta_{i}\right|, i=1,2,3, \ldots$; moreover, from (5.4) we have

$$
n_{\zeta_{i}}\left(t_{i}, \delta\right) \geqslant t_{i}\left|\zeta_{i}\right|^{\rho-1}, \quad i=1,2,3, \ldots
$$

Hence

$$
\sum_{1}^{\infty} \frac{t_{i}}{\left|\zeta_{i}\right|} \leqslant \sum_{1}^{\infty} \frac{n_{\zeta_{i}}\left(t_{i}, \delta\right)}{\left|\zeta_{i}\right|^{\rho}}
$$

Now, if $z_{n}$ is one of the zeros of $F$ contained in $S\left(\frac{1}{2} \delta, R_{1}\right)$ and also in one of the discs, say $C_{i}$, then $\left|z_{n}\right|-\left|\zeta_{i}\right| \leqslant\left|z_{n}-\zeta_{i}\right|<t_{i}<\frac{1}{2}\left|\zeta_{i}\right|$ so $\left|z_{n}\right|<\frac{3}{2}\left|\zeta_{i}\right|$. Hence, from (5.5) and the fact that $K$ covers any point in the plane at most $2 \nu$ times,

$$
\sum_{1}^{\infty} \frac{t_{i}}{\left|\zeta_{i}\right|} \leqslant 2 v\left(\frac{3}{2}\right)^{\rho} \sum \frac{1}{\left|z_{n}\right|^{\rho}},
$$

where the sum on the right-hand side is taken over those zeros of $F$ which are contained in $S\left(\frac{1}{2} \delta, R_{1}\right)$. We proceed to show that this sum is finite.

Let $n$ be a nonnegative integer, and let $b_{n}$ be a positive number satisfying $k^{n} R_{1} \leqslant b_{n}<k^{n+1} R_{1}$ at which

$$
\nu\left(b_{n}\right) \log k \leqslant \int_{k^{n} R_{1}}^{k^{n+1} R_{1}} \nu(t) \frac{d t}{t},
$$

where $\nu(t)$ is the function occurring in Theorem A and $k$ is given by (3.2). The number of zeros of $F$ in

$$
\left\{z: k^{n} R_{1} \leqslant|z|<k^{n+1} R_{1} \text { and }|\arg z| \leqslant \pi-\frac{1}{2} \delta\right\}
$$


is no more than $\nu\left(b_{n}\right) N\left(b_{n}\right)$, where $N\left(b_{n}\right)$ is the number of zeros of $F$ in $\{z$ : $\left.k^{-1} b_{n} \leqslant|z|<k b_{n}\right\}$. Hence, making use of (5.7) we have, for some constant A,

$$
\begin{aligned}
\sum \frac{1}{\left|z_{n}\right|^{\rho}} & \leqslant \sum_{0}^{\infty} \nu\left(b_{n}\right) N\left(b_{n}\right)\left(k^{n} R_{1}\right)^{-\rho} \\
& \leqslant \sum_{0}^{\infty} \nu\left(b_{n}\right) A b_{n}^{\rho}\left(k^{n} R_{1}\right)^{-\rho} \leqslant A k^{\rho} \sum_{0}^{\infty} \nu\left(b_{n}\right) \\
& \leqslant A k^{\rho}(\log k)^{-1} \int_{R_{1}}^{\infty} \nu(t) \frac{d t}{t}<\infty
\end{aligned}
$$

from Theorem A. The sum on the left-hand side of (5.6) is thus finite.

Suppose that $z$ is a point outside $H_{1}$ and satisfying $|z| \geqslant R_{1}$. Then, given any positive number $\varepsilon<\cdot \frac{1}{2}, \int_{0}^{\varepsilon|z|} n_{z}(t, \delta) d t / t<\varepsilon|z|^{\rho}$. This proves Lemma 3 .

6. The behaviour of $f_{1}(z)$. Let $f_{1}(z)$ be the function (2.3). Since

$\log m(r, f)-\cos \pi \rho \log M(r, f) \geqslant \log m\left(r, f_{1}\right)-\cos \pi \rho \log M\left(r, f_{1}\right)$ it follows from (i)' and Kjellberg's Lemma [8, p. 193, formula (21)] that

$$
\int^{\infty}\left|\log m\left(r, f_{1}\right)-\cos \pi \rho \log M\left(r, f_{1}\right)\right| \frac{d r}{r^{\rho+1}}<\infty .
$$

Given a positive number $\varepsilon>0$, it follows from (6.1) and (2.2) that

$$
\log m\left(r, f_{1}\right)>\left(\beta \cos \pi \rho-\frac{1}{2} \varepsilon\right) r^{\rho}
$$

for $r$ outside a set $E=E(\varepsilon)$ of finite logarithmic measure. Hence, for $\delta=\varepsilon / 2 \beta \rho$,

$$
\log \left|f_{1}\left(r e^{i \theta}\right)\right|>\log m\left(r, f_{1}\right)>(\beta \cos \rho \theta-\varepsilon) r^{\rho}
$$

for $\pi \geqslant|\theta| \geqslant \pi-\delta$ and for $r$ outside $E$.

It is well known (see e.g. [12, p. 272]) that

$$
\left|r^{-\rho} \log \right| f_{1}\left(r e^{i \theta}\right)|-\beta \cos \rho \theta| \rightarrow 0
$$

as $r \rightarrow \infty$, uniformly for $|\theta| \leqslant \pi-\delta$. In particular

$$
\left|r^{-\rho} \log \right| f_{1}\left(r e^{i(\pi-\delta)}\right)|-\beta \cos \rho(\pi-\delta)| \rightarrow 0
$$

as $r \rightarrow \infty$. Hence, for $\pi \geqslant|\theta| \geqslant \pi-\delta$ and for sufficiently large $r$

$$
\begin{aligned}
r^{-\rho} \log \left|f_{1}\left(r e^{i \theta}\right)\right| & \leqslant r^{-\rho} \log \left|f_{1}\left(r e^{i(\pi-\delta)}\right)\right| \\
& \leqslant \beta \cos \rho(\pi-\delta)+\frac{1}{2} \varepsilon \\
& <\beta \cos \rho \theta+2 \beta \sin \frac{1}{2} \rho(|\theta|-\pi+\delta)+\frac{1}{2} \varepsilon \\
& <\beta \cos \rho \theta+\varepsilon .
\end{aligned}
$$

Taking (6.2) and (6.4) together, and taking account of (6.3) we obtain 
LEMMA 5. Let $f_{1}(z)$ be the integral function (2.3). Given $\varepsilon>0$

$$
\left|r^{-\rho} \log \right| f_{1}\left(r e^{i \theta}\right)|-\beta \cos \rho \theta|<\varepsilon \quad(-\pi<\theta \leqslant \pi)
$$

for all large $r$ outside $E$, a set of finite logarithmic measure.

From this together with Lemma 1 we deduce

LEMMA 6. Given any $\varepsilon>0$ there exist positive numbers $\delta=\delta(\varepsilon)$ and $r(\varepsilon)$ such that

$$
\int_{0}^{\delta r} n_{-r}\left(t, f_{1}\right) \frac{d t}{t}<\varepsilon r^{\rho}
$$

for all $r>r(\varepsilon)$ lying outside a set $E_{0}$ of finite logarithmic measure, where $n_{-r}\left(t, f_{1}\right)$ is the number of zeros of $f_{1}$ in $(-r-t,-r+t)$. Further, $E_{0}$ is independent of $\varepsilon$ and is a union of disjoint intervals each of which contains more than one point.

We need verify only that $E_{0}$ may be taken to be a union of disjoint intervals each containing more than one point; Lemma 6 certainly holds for some set $E_{1}$ of finite logarithmic measure and some functions $\delta(\varepsilon), r(\varepsilon)$, by Lemma 1.

To this end, given $\varepsilon>0$, let $\eta=\delta\left(\frac{1}{3} \varepsilon\right)$, where $\delta$ is the function known to exist and suppose that $r_{1}$ and $r_{2}$ are two points outside $E_{1}$ with $2 r_{1} \geqslant r_{2}>r_{1}>r\left(\frac{1}{3} \varepsilon\right)$ and such that $f_{1}$ has no zeros in $\left[r_{1}, r_{2}\right]$. Then, for $r_{1}<r<r_{2}$

$$
\begin{aligned}
I & =\int_{0}^{\eta r} n_{-r}\left(t, f_{1}\right) \frac{d t}{t} \\
& =\sum_{1}^{n_{1}} \log \frac{\eta r}{r+x_{n}}+\sum_{1}^{n_{2}} \log \frac{-\eta r}{r+y_{n}},
\end{aligned}
$$

where $x_{1}, \ldots, x_{n_{1}}$ are the zeros of $f_{1}$ in $(-r,-r+\eta r)$ and $y_{1}, \ldots, y_{n_{2}}$ are the zeros of $f_{1}$ in $(-r-\eta r,-r)$. Now

$$
\frac{r}{r+x_{n}}=1-\frac{x_{n}}{r+x_{n}}<1-\frac{x_{n}}{r_{1}+x_{n}}=\frac{r_{1}}{r_{1}+x_{n}},
$$

so, in view of Lemma 1 and Lemma 5

$$
\begin{aligned}
\sum_{1}^{n_{1}} \log \frac{\eta r}{r+x_{n}} & <\sum_{1}^{n_{1}} \log \frac{\eta r_{1}}{r_{1}+x_{n}} \\
& \leqslant \int_{0}^{\eta r_{1}} n_{-r_{1}}\left(t, f_{1}\right) \frac{d t}{t}<\frac{1}{3} \varepsilon r_{1}^{\rho} .
\end{aligned}
$$

Similarly, $-r /\left(r+y_{n}\right)<-r_{2} /\left(r_{2}+y_{n}\right)$, so

$$
\sum_{1}^{n_{2}} \log \frac{-\eta r}{r+y_{n}} \leqslant \int_{0}^{\eta r_{2}} n_{-r_{2}}\left(t, f_{1}\right) \frac{d t}{t}<\frac{1}{3} \varepsilon r_{2}^{\rho}<\frac{2}{3} \varepsilon r_{1}^{\rho} \text {. }
$$


From (6.6), (6.7) and (6.8),

$$
\int_{0}^{\eta r} n_{-r}\left(t, f_{1}\right) \frac{d t}{t}<\varepsilon r_{1}^{\rho}<\varepsilon r^{\rho}
$$

for $r$ in $\left(r_{1}, r_{2}\right)$.

Let $E_{0}$ be the set obtained by removing from $E_{1}$ all points which are limit points both from the left and from the right of the complement of $E_{1}$. Then $E_{0}$ is a union of disjoint intervals each containing more than one point and is contained in $E_{1}$. Moreover, if $r>r\left(\frac{1}{3} \varepsilon\right)$ and $r$ lies outside $E_{0}$, then (6.9) holds, with $\eta=\eta(\varepsilon)=\delta\left(\frac{1}{3} \varepsilon\right)$. This completely proves Lemma 6 .

7. Further consideration of the zeros of $F$. We first observe that the set of intervals the union of which is $E_{0}$ is countable since the logarithmic measure of $E_{0}$ is finite and the logarithmic measure of each interval is positive. We may therefore regard each interval as closed without affecting the value of the logarithmic measure of $E_{0}$. We write $E_{0}=\cup_{1}^{\infty} J_{i}$, where

$$
J_{i}=\left[c_{i}, d_{i}\right], \quad c_{i+1}>d_{i}>c_{i}, \quad i=1,2,3, \ldots
$$

Let $\varepsilon$ be any positive number. With Lemmas 3 and 6 in view, let $\tau=\tau(\varepsilon)=\min \left(\frac{1}{2}, \varepsilon, \delta(\varepsilon)\right.$ ) (where $\delta(\varepsilon)$ is the function of Lemma 6) and let $r_{0}(\varepsilon)$ be a number at least as large as $\max \left(r(\varepsilon), R\left(\tau, \frac{1}{8} \tau\right)\right.$ ) (where $r(\varepsilon), R(x, y)$ are respectively the functions of Lemmas 6 and 3$)$ such that $r_{0}(\varepsilon) \notin E_{0}\left(E_{0}\right.$ is the set of Lemma 6) and for which $d_{i}<\left(1+\frac{1}{4} \tau\right) c_{i}$ whenever $c_{i}>r_{0}(\varepsilon)$. Since $E_{0}$ is of finite logarithmic measure this choice of $r_{0}(\varepsilon)$ is possible.

Define, for $J_{i}=\left[c_{i}, d_{i}\right]$ where $c_{i}>r_{0}(\varepsilon)$,

$$
\begin{aligned}
& B_{i}=\left\{z:|z| \in J_{i}\right\} \cap\left\{z: \pi \geqslant|\arg z|>\pi-\frac{1}{8} \tau\right\}, \\
& D_{i}=\left\{z:|z| \in J_{i}\right\} \cap\left\{z: \pi \geqslant|\arg z|>\pi-\frac{1}{8} \tau\right\} \backslash H_{1}
\end{aligned}
$$

where $H_{1}$ is the exceptional set of Lemma 3 corresponding to $\delta=\frac{1}{8} \tau$. $H_{1}=H_{1}(\tau)=H_{1}(\varepsilon)$.

Let $z$ be any point in $D_{i}$. Then, with $r=|z|$,

$$
\begin{aligned}
I(z) & =\int_{0}^{\tau r} n_{z}(t, F) \frac{d t}{t} \\
& =\log \frac{(\tau r)^{m+n+p+q}}{\Pi_{1}^{m}\left|z-a_{j}\right| \Pi_{1}^{n}\left|z-b_{j}\right| \Pi_{1}^{p}\left|z-u_{j}\right| \Pi_{1}^{q}\left|z-v_{j}\right|},
\end{aligned}
$$

where the $a_{j}, b_{j}, u_{j}, v_{j}$ are zeros of $F$ in $|\zeta-z|<\tau r$ which are respectively in $B_{i}$, in $\left\{z:|z| \in J_{i}\right\} \backslash B_{i}$, in $|z|<c_{i}$ and in $|z|>d_{i}$. Then we have, recalling Lemma 3 and Lemma 6 , 


$$
\begin{aligned}
I(z) \leqslant & \log \frac{(\tau r)^{m}}{\Pi_{1}^{m}\left|z-a_{j}\right|}+\log \frac{(\tau r)^{n}}{\prod_{j=1}^{n}\left(r-\left|u_{j}\right|\right)}+\log \frac{(\tau r)^{p}}{\prod_{j=1}^{p}\left(\left|v_{j}\right|-r\right)} \\
& +\int_{0}^{\tau r} n_{z}\left(t, F, \frac{1}{8} \tau\right) \frac{d t}{t} \\
\leqslant & \log \frac{(\tau r)^{m}}{\Pi_{1}^{m}\left|z-a_{j}\right|}+\log \frac{\left(\tau c_{i}\right)^{n}}{\Pi_{j=1}^{n}\left(c_{i}-\left|u_{j}\right|\right)}+\log \frac{\left(\tau d_{i}\right)^{p}}{\prod_{j=1}^{p}\left(\left|v_{j}\right|-d_{i}\right)} \\
& +\int_{0}^{\tau r} n_{z}\left(t, F, \frac{1}{8} \tau\right) \frac{d t}{t}
\end{aligned}
$$

$$
\begin{aligned}
& <\log \frac{(\tau r)^{m}}{\Pi_{1}^{m}\left|z-a_{j}\right|}+\int_{0}^{\tau c_{i}} n_{-c_{i}}\left(t, f_{1}\right) \frac{d t}{t}+\int_{0}^{\tau d_{i}} n_{-d_{i}}\left(t, f_{1}\right) \frac{d t}{t} \\
& \quad+\int_{0}^{\tau r} n_{z}\left(t, F, \frac{1}{8} \tau\right) \frac{d t}{t} \\
& <\log \frac{(\tau r)^{m}}{\Pi_{1}^{m}\left|z-a_{j}\right|}+\int_{0}^{\delta(\varepsilon) c_{i}} n_{-c_{i}}\left(t, f_{1}\right) \frac{d t}{t}+\int_{0}^{\delta(\varepsilon) d_{i}} n_{-d_{i}}\left(t, f_{1}\right) \frac{d t}{t} \\
& \quad+\int_{0}^{\tau r} n_{z}\left(t, F, \frac{1}{8} \tau\right) \frac{d t}{t} \\
& <\log \frac{(\tau r)^{m}}{\Pi_{1}^{m}\left|z-a_{j}\right|}+\varepsilon\left[c_{i}^{\rho}+d_{i}^{\rho}\right]+\tau r^{\rho} \\
& <\log \frac{(\tau r)^{m}}{\Pi_{1}^{m}\left|z-a_{j}\right|}+4 \varepsilon r^{\rho} .
\end{aligned}
$$

Now, $B_{i}$ is contained in a rectangle the sides of which have length $\frac{1}{4} \tau d_{i}$ $<\frac{3}{8} \tau c_{i}$ and $d_{i}-c_{i} \cos \frac{1}{8} \tau$. Hence for any point $z$ in $D_{i}$ the circle $|\zeta-z|<$ $\tau|z|$ contains all of $B_{i}$ and so all the zeros of $F$ in $B_{i}$ (i.e. all the $a_{j}$ ) appear in (7.1). We can thus apply Cartan's Lemma [3, p. 75] to estimate (7.1) and obtain

$$
\prod_{1}^{m}\left|z-a_{j}\right| \geqslant\left\{\tau d_{i} \exp \left(-\frac{\varepsilon d_{i}^{\rho}}{m}\right)\right\}^{m}
$$

outside a set of at most $m$ discs $C_{j}^{\prime}, j=1,2, \ldots, m$, the sum of the radii of which is at most $A=2 e \tau d_{i} \exp \left(-\varepsilon d_{i}^{p} / m\right)$. Hence, for all $z$ in $D_{i}$ outside these discs we have, from (7.1),

$$
\int_{0}^{\tau r} n_{z}(t, F) \frac{d t}{t}<4 \varepsilon r^{\rho}+\log \left(\frac{r}{d_{i}}\right)^{m}+\varepsilon d_{i}^{\rho}<6 \varepsilon r^{\rho} .
$$

We must have $A<2 e\left(d_{i}-c_{i}\right)$. For suppose that $A>2 e\left(d_{i}-c_{i}\right)$. Then 


$$
\begin{aligned}
I\left(\varepsilon, d_{i}\right) & =\int_{0}^{\delta(\varepsilon) d_{i}} n_{-d_{i}}\left(t, f_{1}\right) \frac{d t}{t} \geqslant \int_{0}^{\tau d_{i}} n_{-d_{i}}\left(t, f_{1}\right) \frac{d t}{t} \\
& \geqslant \log \frac{\left(\tau d_{i}\right)^{m}}{\prod_{j=1}^{m}\left(d_{i}-\left|a_{j}\right|\right)} \\
& \geqslant \log \frac{\left(\tau d_{i}\right)^{m}}{\left(d_{i}-c_{i}\right)^{m}} \\
& >\log \frac{\left(2 e \tau d_{i}\right)^{m}}{A^{m}}=\varepsilon d_{i}^{\rho},
\end{aligned}
$$

a contradiction, since $I\left(\varepsilon, d_{i}\right) \leqslant \varepsilon d_{i}^{\rho}, d_{i}$ being a boundary point of $E_{0}$. Hence

$$
A \leqslant 2 e\left(d_{i}-c_{i}\right) \text {. }
$$

Suppose that $C_{j}^{\prime}$ has radius $t_{j}^{\prime}$ and centre $\zeta_{j}^{\prime}, j=1,2, \ldots, m$.

$$
\sum_{j=1}^{m} \frac{t_{j}^{\prime}}{\left|\zeta_{j}^{\prime}\right|} \leqslant \frac{1}{c_{i}} \sum_{j=1}^{m} t_{j}^{\prime} \leqslant 2 e\left(\frac{d_{i}-c_{i}}{c_{i}}\right) .
$$

Also, since $d_{i}<\left(1+\frac{1}{4} \tau\right) c_{i}<2 c_{i}$ and since, for $x \geqslant 1, \log x \geqslant(x-1) / x$,

$$
\frac{d_{i}-c_{i}}{c_{i}}=\frac{d_{i}}{c_{i}} \frac{d_{i}-c_{i}}{d_{i}}<2 \log \frac{d_{i}}{c_{i}},
$$

so

$$
\sum_{j=1}^{m} \frac{t_{i}^{\prime}}{\left|\zeta_{j}^{\prime}\right|}<4 e \log \frac{d_{i}}{c_{i}}
$$

We are thus able to prove

LEMMA 7. Let $\varepsilon$ be any positive number, and let $\tau=\min \left(\frac{1}{2}, \varepsilon, \delta(\varepsilon)\right)$, where $\delta(\varepsilon)$ is the function of Lemma 6 . Let $r_{0}(\varepsilon)$ be a positive number greater than $\max \left(r(\varepsilon), R\left(\tau, \frac{1}{8} \tau\right)\right)$ such that $r_{0}(\varepsilon) \notin E_{0}$ and for which $d_{i}<\left(1+\frac{1}{4} \tau\right) c_{i}$ whenever $c_{i}>r_{0}(\varepsilon)$, where $r(\varepsilon), R\left(\tau, \frac{1}{8} \tau\right)$ are respectively the functions of Lemmas 6, 3, and $E_{0}$ is the set of Lemma 6. Then for all $z$ in

$$
T\left(\frac{1}{8} \tau, r_{0}(\varepsilon)\right)=\left\{z:|z| \geqslant r_{0}(\varepsilon) \text { and } \pi \geqslant|\arg z| \geqslant \pi-\frac{1}{8} \tau\right\}
$$

we have, with $|z|=r$,

$$
\int_{0}^{\tau r} n_{z}(t, F) \frac{d t}{t}<6 \varepsilon r^{\rho}
$$

except when $z$ belongs to an E-set, $\mathrm{H}_{2}=\mathrm{H}_{2}(\varepsilon)$.

Suppose first that $z$, in $T\left(\frac{1}{8} \tau, r_{0}(\varepsilon)\right)$, lies in $\cup\left\{z:|z| \in J_{i}\right\}$, where the union is over those $J_{i}=\left[c_{i}, d_{i}\right]$ for which $c_{i}>r_{0}(\varepsilon)$. Then for all $z$ outside $H_{1}$, the $E$-set of Lemma 3, and outside a set of discs centres $\zeta$, radii $t$ for which 


$$
\sum \frac{t}{|\zeta|}<4 e \sum \log \frac{d_{i}}{c_{i}}<4 e \log \text { meas } E_{0}<\infty,
$$

(7.5) holds. This follows from (7.2) and (7.4).

Suppose next that $z$, in $T\left(\frac{1}{8} \tau, r_{0}(\varepsilon)\right)$, lies outside $\cup\left\{z:|z| \in J_{i}\right\}$. Then, with $|z|=r$, we have from Lemma 6

$$
\int_{0}^{\tau r} n_{z}(t, F) \frac{d t}{t} \leqslant \int_{0}^{\delta(\varepsilon) r} \eta_{-r}\left(t, f_{1}\right) \frac{d t}{t}<\varepsilon r^{\rho} .
$$

(7.5) thus holds for $z$ in $T\left(\frac{1}{8} \tau, r_{0}(\varepsilon)\right)$ outside an $E$-set, and Lemma 7 is proved. We prove

LEMMA 8. Let $\varepsilon$ be any positive number and let $\sigma=\sigma(\varepsilon)=\frac{1}{32} \tau(\varepsilon)$, where $\tau(\varepsilon)$ is the function of Lemma 7. There exists a number $r_{1}(\varepsilon)$ and an E-set, $H_{3}=H_{3}(\varepsilon)$, such that

$$
\int_{0}^{\sigma r} n_{z}(t, F) \frac{d t}{t}<6 \varepsilon r^{\rho}
$$

whenever $|z|=r>r_{1}(\varepsilon)$ and $z$ lies outside $H_{3}$.

For $z$ in $T\left(\frac{1}{8} \tau, r_{0}(\varepsilon)\right)$ and outside $H_{2}(\varepsilon),(7.6)$ certainly holds, by Lemma 7 .

Consider $z$ outside $T\left(\frac{1}{8} \tau, r_{1}(\varepsilon)\right)$, where $r_{1}(\varepsilon)=\max \left(r_{0}(\varepsilon), R\left(\frac{1}{32} \tau, \frac{1}{8} \tau\right)\right)$. Let $H_{4}(\varepsilon)$ be the $E$-set $H_{1}\left(\frac{1}{8} \tau\right)$ of Lemma 3. Then, with $\sigma=\frac{1}{32} \tau$ and $r=|z|$, and $z$ outside $H_{4}(\varepsilon)$, we have from Lemma 3

$$
\int_{0}^{\sigma r} n_{z}\left(t, F, \frac{1}{8} \tau\right) \frac{d t}{t}<\sigma r^{\rho}<\varepsilon r^{\rho} .
$$

But for $0<t<\frac{1}{32} \tau r$ and $r \geqslant R\left(\frac{1}{32} \tau, \frac{1}{8} \tau\right), n_{z}(t, F)=n_{z}\left(t, F, \frac{1}{8} \tau\right)$ for $z$ outside $T\left(\frac{1}{8} \tau, r_{1}(\varepsilon)\right)$. Hence $\int_{0}^{o r} n_{z}(t, F) d t / t<\varepsilon r^{\rho}$ for $z$ outside $T\left(\frac{1}{8} \tau, r_{1}(\varepsilon)\right)$ and outside $H_{4}(\varepsilon)$, with $|z|=r>R\left(\frac{1}{32} \tau, \frac{1}{8} \tau\right)$.

Lemma 8 then follows with $H_{3}(\varepsilon)=H_{2}(\varepsilon) \cup H_{4}(\varepsilon)$.

The following is an immediate consequence of Lemma 8.

Lemma 9. Let $\varepsilon$ be any positive number. There exist positive numbers $\alpha(\varepsilon)$, $r_{2}(\varepsilon)$ and an $E$-set $H_{5}$, independent of $\varepsilon$, such that

$$
\int_{0}^{\alpha(\varepsilon) r} n_{z}(t, F) \frac{d t}{t}<\varepsilon r^{\rho}
$$

when $r=|z|>r_{2}(\varepsilon)$ and $z$ lies outside $H_{5}$.

For $z$ such that $r=|z|>r_{1}\left(\frac{1}{6}\right)$ and $z$ lies outside $H_{3}\left(\frac{1}{6}\right)$, where $r_{1}$ and $H_{3}$ are as in Lemma 8, we have, with $\sigma=\sigma\left(\frac{1}{6}\right), \int_{0}^{o r} n_{z}(t, F) d t / t<r^{p}$. Given any integer $n>1$, suppose that $H_{3}(1 / 6 n)$ is covered by the discs $C_{i}(n)$, radii $t_{i}(n)$ and centres $\zeta_{i}(n), i=1,2,3, \ldots$ Let $i_{0}=i_{0}(n)$ be the smallest integer such that 


$$
\sum_{i=i_{0}}^{\infty} \frac{t_{i}(n)}{\left|\zeta_{i}(n)\right|} \leqslant 2^{-n} \sum_{i=1}^{\infty} \frac{t_{i}(1)}{\left|\zeta_{i}(1)\right|}, \quad n=2,3, \ldots
$$

Let $r_{2}(1)=r_{1}\left(\frac{1}{6}\right)$ and, supposing $r_{2}(m)$ defined, $m \geqslant 1$, let $r_{2}(m+1)$ be the smallest number which is no less than $\max \left\{r_{2}(1 / m)+1, r_{1}(1 / 6(m+1))\right\}$ and such that

$$
C_{i}(m+1) \subset\left\{z:|z| \leqslant r_{2}(1 /(m+1))\right\}, \quad i=1,2, \ldots, i_{0}(m+1)-1 .
$$

Let $H_{4}$ be given by

$$
H_{4}=\left\{\bigcup_{1}^{\infty} C_{i}(1)\right\} \cup\left\{\bigcup_{n=2}^{\infty} \bigcup_{i=i_{0}(n)}^{\infty} C_{i}(n)\right\} .
$$

From (7.7), $H_{4}$ is an $E$-set.

Given any number $\varepsilon, 0<\varepsilon<1$, let $m$ be the integer such that

$$
\frac{1}{m+1}<\varepsilon \leqslant \frac{1}{m}
$$

define $r_{2}(\varepsilon)=r_{2}(1 /(m+1)), \alpha(\varepsilon)=\sigma(1 / 6(m+1))$. Let $\varepsilon$ be any positive number, $0<\varepsilon<1$, and let $z$ be outside $H_{4}$ and such that $r=|z|>r_{2}(\varepsilon)$. Then, if $m$ is the integer satisfying (7.8), $z$ lies outside $H_{3}(1 / 6(m+1))$ and $r=|z|>r_{1}(1 / 6(m+1))$ so by Lemma 8 ,

$$
\begin{aligned}
\int_{0}^{\alpha(\varepsilon) r} n_{z}(t, F) \frac{d t}{t} & =\int_{0}^{\sigma}\left(\frac{1}{6(m+1)}\right)^{\prime} n_{z}(t, F) \frac{d t}{t} \\
& <\frac{1}{m+1} r^{\rho}<\varepsilon r^{\rho} .
\end{aligned}
$$

Lemma 9 is thus proved.

8. Completion of the proof of Theorem 1. By Lemma 1 and Lemma 9,

$$
\left|r^{-\rho} \log \right| F\left(r e^{i \theta}\right)|-\beta \cos \rho \theta| \rightarrow 0
$$

as $z=r e^{i \theta}$ tends to infinity outside $H_{4}$. From (8.1) and Lemma 2, Theorem 1 follows.

9. A counterexample. Let $f(z)$ be an integral function with real negative zeros. In [11] Titchmarsh proves that if

$$
\varlimsup_{r \rightarrow \infty} \frac{\log |f(r)|}{r^{\rho}}=A \quad(0<A<\infty)
$$

for some $\rho$ such that $0<\rho<1$ then

(i) $\varlimsup_{r \rightarrow \infty} \log |f(-r)| / r^{\rho}=A \cos \pi \rho$; and

(ii) given $\varepsilon>0$,

$$
\log |f(-r)|>(A \cos \pi \rho-\varepsilon) r^{\rho}
$$

for all $r$ outside a set of linear density zero. 
We shall show that the exceptional set of (ii) cannot be replaced with a set of finite logarithmic measure by constructing an integral function satisfying (9.1) for which (9.2) fails for some $\varepsilon>0$ on a set of infinite logarithmic measure. The construction depends on Lemma 1.

Let $A$ be any fixed positive number. Let $\left(R_{m}\right)$ be an increasing sequence of positive numbers, let $\eta_{m}=(\log m)^{-1}$ and let $\delta_{m}=m^{-1 / 2}, m=2,3 \ldots$ Let $f(z)$ be an integral function with real negative zeros for which the counting function $n(r, f)$ satisfies

$$
n(r, f) \sim A r^{\rho}
$$

We introduce an integral function $g(z)$ obtained from $f(z)$ by placing $1+$ [ $\eta_{m} R_{m}^{\rho}$ ] additional zeros at $-R_{m}$. It is clear that the sequence $\left(R_{m}\right)$ may be chosen sparsely enough that $n(r, g)$, the counting function of the zeros of $g(z)$, satisfies $n(r, g) \sim A r^{\rho}$.

With Lemma 1 in view let us consider, for $R_{m}<r<(1-1 / m)^{-1} R_{m}$,

$$
\int_{0}^{\delta_{m} r} \frac{n_{-r}(t, g)}{t} d t
$$

Since $r-R_{m}<r-r\left(1-m^{-1}\right)<\delta_{m} r$, each zero at $-R_{m}$ contributes $\log \left\{\delta_{m} r / r-R_{m}\right\}$ to the integral (9.3). Hence we have, for $R_{m}<r<$ (1$1 / m)^{-1} R_{m}$

$$
\begin{aligned}
\int_{0}^{\delta_{m} r} \frac{n_{-r}(t, g)}{t} d t & \geqslant \eta_{m} R_{m}^{\rho} \log \left\{\frac{\delta_{m} r}{r-R_{m}}\right\} \\
& \geqslant \eta_{m} R_{m}^{\rho} \log \left(m \delta_{m}\right)=\frac{1}{2} R_{m}^{\rho} \\
& >\frac{1}{4} r^{\rho}
\end{aligned}
$$

for all large $m$, taking account of the definitions of $\eta_{m}$ and $\delta_{m}$. Further

$$
E=\bigcup_{m=3}^{\infty}\left\{r: R_{m}<r<\left(1-\frac{1}{m}\right)^{-1} R_{m}\right\}
$$

is a set of infinite logarithmic measure.

Now we appeal to Lemma 1 to conclude that there must be a number $\varepsilon>0$ such that

$$
\log |f(-r)|<(A \cos \pi \rho-\varepsilon) r^{\rho}
$$

for all large $r$ in $E$. For suppose that there were a sequence $\left(r_{n}\right)$ tending to infinity through $E$ such that

$$
\log \left|f\left(-r_{n}\right)\right| \geqslant(A \cos \pi \rho-o(1)) r_{n}^{\rho} .
$$

From (i) of Titchmarsh's result, then,

$$
\log \left|f\left(-r_{n}\right)\right|=(A \cos \pi \rho+o(1)) r_{n}^{\rho}
$$


and so, from Lemma 1 , there must exist $\delta>0$ such that

$$
\int_{0}^{\delta r_{n}} \frac{n_{-r_{n}}(t, g)}{t} d t<\frac{1}{4} r_{n}^{\rho}
$$

for all large $n$, which contradicts (9.4). (9.5) thus holds for all large $r$ in $E$.

Theorem 1 is an improved version of a result which forms part of a thesis submitted for the degree of $\mathrm{Ph} . \mathrm{D}$ at the University of London. It is a pleasure to express my gratitude to Professor W. K. Hayman of Imperial College, London, for his generous advice and encouragement.

\section{REFERENCES}

1. J. M. Anderson, Asymptotic properties of integral functions of genus zero, Quart. J. Math. Oxford Ser. (2) 16 (1965), 151-164.

2. V. Azarin, Generalization of a theorem of Hayman on subharmonic functions in an n-dimensional cone, Amer. Math. Soc. Transl. (2) 80 (1969), 119-138.

3. M. L. Cartwright, Integral functions, Cambridge Univ. Press, London and New York, 1956.

4. M. Essén, A generalization of the Ahlfors-Heins theorem, Trans. Amer. Math. Soc. 142 (1969), 331-344.

5. M. Essén and J. Lewis, The generalized Ahlfors-Heins theorem in certain d-dimensional cones, Math. Scand. 33 (1973), 113-129.

6. P. C. Fenton, The distribution of the Riesz mass of certain subharmonic functions, Ark. Mat. 14 (1976), 259-276.

7. W. K. Hayman, Slowly growing integral and subharmonic functions, Comment. Math. Helv. 34 (1960), 75-84.

8. B. Kjellberg, On the minimum modulus of entire functions of order less than one, Math. Scand. 8 (1960), 189-197.

9. T. A. Kolomiiceva, The asymptotic behaviour of an entire function with regular root distribution, Teor. Funkcii Funkcional. Anal. i Priložen. Vyp. 15 (1972), 35-43. (Russian)

10. N. S. Landkof, Foundations of modern potential theory, Springer-Verlag, Berlin and New York, 1972.

11. E. C. Titchmarsh, On integral functions with real negative zeros, Proc. London Math. Soc. (2) 26 (1927), 185-200.

12. , The theory of functions, Oxford Univ. Press, London and New York, 1939.

Department of Mathematics, University of Otago, Dunedin, New Zealand 